\title{
Effects of Tai Chi Intervention on Perceived Stress, Anxiety, and Sleep in College Students
}

\author{
Weiyun Chen ${ }^{1}$, Shouwen $\mathrm{Yu}^{2}$, Delang Xiong ${ }^{3}$ \\ ${ }^{1}$ School of Kinesiology, University of Michigan, Ann Arbor, MI, USA \\ ${ }^{2}$ East China University of Political Science and Law, Shanghai, China \\ ${ }^{3}$ East China Normal University, Shanghai, China \\ Email: *chenwy@umich.edu
}

How to cite this paper: Chen, W. Y., Yu, S. W., \& Xiong, D. L. (2020). Effects of Tai Chi Intervention on Perceived Stress, Anxiety, and Sleep in College Students. $A d$ vances in Physical Education, 10, 54-67. https://doi.org/10.4236/ape.2020.101006

Received: January 2, 2020

Accepted: February 18, 2020

Published: February 21, 2020

Copyright ( 2020 by author(s) and Scientific Research Publishing Inc. This work is licensed under the Creative Commons Attribution-NonCommercial International License (CC BY-NC 4.0). http://creativecommons.org/licenses/by-nc/4.0/ (c) (i) (s) Open Access

\begin{abstract}
Background: Previous studies on effects of Tai Chi on physical and mental health among college students have lacked an objective measure of sleep quality. This study will address the gaps in the current literature. Purpose: This study examined the effects of 16-week Tai Chi lessons on perceived stress, anxiety, and sleep quality measured with the ActiGraph activity monitor in college students. Methods: Participants were forty-five college students (14 males and 31 females) with mean age of 19 yrs. ( $\mathrm{SD}=0.548 \mathrm{yrs}$.) who enrolled in one, 90 -min Tai Chi lesson per week for 16 weeks during a fall semester of 2016 at one major public university in Shanghai, China. One week prior to and after the 16-week Tai Chi lessons, the students completed the Perceived Stress Scale (PSS), the Generalized Anxiety Disorder 7-item (GAD-7) Scale. In addition, they wore the ActiGraph Gt3X-BT activity monitor day and night for seven consecutive days to objectively measure their sleep time and quality. Results: The paired t-tests showed no significant changes in the perceived stress and anxiety from the pre- to the post-test ( $t=-0.429, d f=37, p=0.670 ; t=-1.605, d f=37, p=$ $0.117)$. The ANOVA with repeated measures revealed no significant main effect of gender, time, and the interaction of them in the perceived stress and anxiety. The paired t-test yielded a significant change in sleep time $(t=2.48, d t$ $=37, p=0.017)$. There was no significant main effect of gender and the interaction of time with gender. The paired $t$-test showed no significant differences in sleep quality over times. Conclusion: Learning and performing one, 90-min Tai Chi lesson weekly for 16 weeks helped the students maintain a manageable level of the perceived stress and anxiety and meet the recommended sleep time and to have a high level of sleep quality while experiencing a moderate level of stress and anxiety over times.
\end{abstract}




\section{Keywords}

Stress, Anxiety, Sleep Time, Sleep Quality, Tai Chi

\section{Introduction}

Mental health problems and sleep deprivation are major public health concerns among college students. College students are more likely to be at-risk for having mental health problems due to facing a myriad of stressors including academic challenges, peer pressures, social relationships, and financial issues as well as lack of sleep quantity and quality. According to the 2018 American College Health Association (ACHA) National College Health Assessment of 88,178 students at 140 universities in USA, the prevalence and severity of mental health problems faced by college students were reaching an alarming rate. When self-rating an overall level of stress they experienced within the last 12 months, $34.3 \%$ of the students indicated that they experienced average stress, $44.9 \%$ more than average stress, and $12.7 \%$ tremendous stress. In addition, $32.9 \%$ of the students experienced sleep difficulties within the last 12 months (ACHA, 2018). Furthermore, college students in two studies $(\mathrm{n}=1629, \mathrm{n}=1125)$ reported that emotional and academic stresses were the most significant risk factors contributing to their poor sleep quality, accounting for $13.5 \%$ and $24 \%$ of the variance in the global sleep score, respectively (Chung \& Cheung, 2008; Lund, Reider, Whiting, \& Prichard, 2010). College students who are chronically suffering from mental health problems and poor sleep quality and deprivation are susceptible to experiencing academic struggling, withdrawing from schools, attempting and/or committing suicide. However, only $22.1 \%$ of students suffering from anxiety, $18.1 \%$ of students with depression, and $9.1 \%$ of students having insomnia and other sleep disorders were treated by professionals within the last 12 months (ACHA, 2018). Due to the severity of mental health problems in college students and their reluctance to seek and receive consistent professional services and help, there is an urgent need for exploring an alternative approach to a primary prevention and treatment of mental health problems.

Regular physical activity is a cost-effective and alternative approach to enhance physical and mental health outcomes (Li et al., 2012; Li et al., 2018). Tai Chi is known as "moving meditation", mind-body exercises with low-to-moderate intensity. Tai Chi has been increasingly practiced to promote and obtain benefits of physical and mental health in general population. Tai Chi, regardless of various styles and forms, consists of sequential and continuous coordinated whole-body movements. Tai Chi is performed with a slightly semi-squat position in a controlled and balanced manner and is transited from one movement to another in a smooth flow. Simultaneously, Tai Chi is practiced with cognitively concentrating on each eye-hand-trunk-leg-foot coordinated movement in a gentle and smooth motion while maintaining balanced posture synchronized with a rhythmic breath- 
ing in and out pattern (Li et al., 2012; Li et al., 2018).

Given the nature of the meditative mind-body exercise, effects of Tai Chi on physical and mental health have been increasingly investigated in older adults and chronic conditioned population. For example, a systematic review of 40 studies in effects of Tai Chi on stress and anxiety in community dwelling healthy older adults and health conditioned older adults as well as patients with chronic diseases was conducted (Wang et al., 2010). Twenty-one of 33 randomized and nonrandomized studies found that practicing Tai Chi from one hour to one year (20 - 120 minutes per session, 1 to 4 times per week) significantly reduced levels of stress and anxiety measures in healthy older adults, older adults with hip or knees osteoarthritis, older adults with cardiovascular disease risk factors, and patients with HIV (Wang et al., 2010). In addition, several systematic reviews reported Tai Chi is the effective mind-body exercise for promoting physical and mental health in the middle-aged adult and older adult population (Wang et al., 2010; Zheng et al., 2015).

However, few studies investigated the effects of Tai Chi on physical and mental health in college students. To the best of our knowledge, only four studies of Tai Chi with college students were found. Wang, Taylor, Pearl, \& Chang (2004) examined the effects of 3-month 24-form Tai Chi (one hour two times per week) on thirty college students' physical and mental health measured with SF-36 v2 at pre- and post-test. Paired t-test indicated significant positive changes in bodily pain, general health, role mental/emotion function, vitality, and perceptions of mental health from the pre-test to the post-test. In a study of 35 college students participating in 15-week semester Tai Chi classes (two 50 min of Chen-Style Tai Chi classes per week), Caldwell, Harrison, Adams, \& Triplett (2009) reported that Tai Chi group showed positive trend toward an improvement in sleep quality measured with Pittsburgh Sleep Quality Index (PSQI). The Tai Chi group also exhibited a significant improvement in relaxation, positive energy, and negative mood sub-scales of the Four Dimensional Mood Scale over the course of a semester. Similarly, Caldwell, Emery, Harrison, \& Greeson (2011) used quasi-experimental design to study the effects of 15-week course of Chen-Style Tai Chi on well-being and sleep quality in 76 college students, compared with 132 college students (control group) receiving a 15-week course of special recreation class. The Tai Chi group showed significant decreased scores in sleeping disturbance and perceived stress score as well as significant improvement in four mood dimensions over the course of the semester, compared with the special recreation group. In contrast, a study with a randomized controlled trial design investigated effects of a 12-week 24-form Tai Chi intervention (five $60 \mathrm{~min}$ of Tai Chi sessions per week) on physical and mental health in 206 college students (Zheng et al., 2015). The results showed that the intervention students $(n=103)$ significantly improved their flexibility and balance compared to the control group ( $n=103$ ) receiving usual activities. However, the 12-week Tai Chi practices did not produce significant positive intervention effects on self-report measure of stress and sleep quality (Zheng et al., 2015). 
Given the scarcity of study on Tai Chi with college students and its effect on gender as well as lack of objective measure of sleep quality in previous Tai Chi studies, this study will address the gaps in the current literature. Thus, the purpose of this study was to examine the effects of 16-week Tai Chi classes on perceived stress, anxiety, and sleep quality measured with the ActiGraph activity monitor in college students and its effects on gender. Specially, we focused on examining three research questions: 1) would the 16-week Tai Chi classes significantly improve students' perceived stress level from the pre-test to the post-test and had any gender effects? 2) would the 16-week Tai Chi classes significantly improve students' anxiety from the pre-test to the post-test and had gender effects? and 3) would the 16-week Tai Chi classes significantly improve students' sleep time and quality from the pre-test to the post-test and had gender effects? The significance of the study lies in providing further empirical support for using the non-pharmaceutical approach to promote physical and mental health in college students.

\section{Methods}

\subsection{Research Design and Participants}

This study used a pre-test and post-test within subject design. All students who enrolled in the two Tai Chi classes offered in the fall semester of 2016 at one major public university in Shanghai were invited to participate in this study using a convenient purpose sample method. The physical education teacher informed the students the research purpose, intervention, assessments, voluntary participation, and confidentiality during a regular physical education class as well as disturbing the content form to the students. Forty-five students from the two classes (14 males and 31 females) with mean age of $19 \mathrm{yrs}$. ( $\mathrm{SD}=0.548 \mathrm{yrs}$.) indicated their voluntary participation in this study by returning their signed the consent forms to the PE teacher during a signed week. Prior to data collection, the University Institutional Review Board (IRB)-Health Sciences and Behavioral Sciences (HSBS) approved the project (HUM00102146).

One week prior to the intervention, the participating students completed three outcome measures at pre-test. Then, the students attended a 16-week Tai Chi intervention. Each of the two Tai Chi classes met once a week for 90 minutes over 16 weeks. The two Tai Chi classes were taught by one experienced certified physical education teacher who had five-year experience teaching Tai Chi classes. In the two Tai Chi classes, the instructor taught 14-form Dragon-Snake Tai Chi by using the structured lesson format. Each structured lesson consists of warm-ups (i.e., stretching exercises), basic semi-squat postures in stationary and with traveling (i.e., horse-step standing), and learning one new form of Tai Chi, and reviewing and practicing previous learned forms of Tai Chi. The instructor organized the students into small groups of 5 - 6 students for group practicing Tai-chi forms learned from the classes with their scheduled times: five times per week after the class. Immediately after the intervention, the participating students completed the three outcome measures again at post-test. 


\subsection{Data Collection}

Participating students in the two classes completed the questionnaires via online Star Questionnaire app at the beginning and the end of the semester. In addition, one week prior to and one week after the 16-week Tai Chi intervention, students wore the ActiGraph activity monitors for seven consecutive days and nights.

Perceived Stress Scale (PSS). The 10-item PSS was designed to measure how often an individual had stressful life situations in the past month using a 5-point rating scale anchored from $(0=$ never, $1=$ almost never, $2=$ sometimes, $3=$ fairly often, and $4=$ very often) (Cohen, Kamarck, \& Mermelstein, 1983). The PSS is a widely used, reliable, and valid psychological instrument used in stress reduction intervention studies with higher scores indicating greater stress. Items $4,5,7$, and 8 were reversely coded. In this study, the students used the Chinese version of PSS (Leung, Lam, \& Chan, 2010) to self-rate how often they felt stressful in a certain way for each question. The Cronbach's alpha coefficient for the total scale was 0.86 at the pre-test and 0.83 at the post-test.

The Generalized Anxiety Disorder 7-item (GAD-7) Scale. The GAD-7 was designed to self-assess how often an individual experienced each of seven problems in the last two weeks with the 4 -point rating scales $(0=$ not at all, $1=$ several days, 2 = more than half the days, 3 = nearly every day) (Spitzer, Kroenke, Williams, \& Lowe, 2006). The GAD-7 is a widely used gold-standard measurement tool for self-assessing generalized anxiety disorder (Spitzer, et al., 2006). In this study, the students used the Chinese version of the GAD-7 ( He, Li, Qian, Cui, \& Wu, 2010) to self-rate how often they had the symptoms of the seven problems in the past two weeks. The Cronbach's alpha value was 0.78 at the pre-test and 0.88 at the post-test.

ActiGraph activity monitor measure of sleep quality. The ActiGraph GT3X-BT activity monitor has been validated for sleep-wake estimation against polysomnography (Lee, Byun, Keill, Dinkel, \& Seo, 2018). To objectively measure the students' sleep quality and time, the students were asked to wear the ActiGraph wGT3X-BT monitor on their waist for seven consecutive days and nights. In addition, they recorded their times to go to bed per night and times to get up per morning using the daily sleeping log. They were only allowed to remove the device when bathing and during other water activities. Prior to distributing the activity monitor to each student, the trained investigator initialized each device with the ActiLife data analysis software for each participant. Each device was set the starting time: Wednesday at 9:00 am and the stopping time: next Wednesday at 8:59 am. When distributing the devices to each participant, the trained investigator explained and demonstrated how to wear it and what wearing protocols were. In addition, the investigator distributed and explained how to record the daily sleep log in terms of times to go to bed per night and times to get up next morning. One week later, the students returned their ActiGraph activity monitor along with the daily sleeping log to the investigator. Then, each device's sleeping variables were analyzed using the Sleep of the ActiLife 6.11 .3 by defining the 
participant's sleeping time each night and waking up time next morning using their recorded daily sleeping log. Next, each participant's total sleep time, sleep efficiency, wake after sleep onset (WASO) (min), \# of awakenings, and average wakening (min) were calculated using the sleep algorithm: Cole-Kripke of the ActiLife software. The total sleep time was the sum of the number of sleep minutes during the sleep period. Sleep-efficiency was the percentage of time spent in bed that the person was asleep. WASO was the number of minutes until the first awakening after sleep onset.

\subsection{Data Analysis}

Among 45 participating students, seven students were excluded from the final data analysis because of missing one of the three outcome measures either at the pre-test, the post-test, or both. The missing data were screened by using a listwise deletion method. The final data set consisted of 38 students ( 27 females vs. 11 males) for data analysis. The composite scores of the PSS and of the GAD-7 scale were calculated. Descriptive statistics were computed for the PSS and the GAD-7 scale and for the five sleep variables, including the total sleep time (min), sleep efficiency, WASO (min), \# of awakenings, and average awakening (min) for the total sample and by gender. To examine if there were any significant changes in each of the variables over times within the total sample, the paired t-tests were performed for the total sample. The percent improvement (size effect) from the pre-test to the post-test for the total sample was calculated based on the formula: percentage of change $=\left(\frac{\bar{X}_{2}-\bar{X}_{1}}{\bar{X}_{1}}\right) \times 100$. To examine if there are any significant changes in each of the variables from the pre-test to the post-test between females and males, a $2 \times 2$ mixed-design analysis of variance (ANOVA) with repeated measures were performed with time (pre-test vs. post-test) as the within-subject factor and gender (females vs. males) as the between-subject factor. When the assumption of sphericity was violated, the Greenhouse-Geyser correction was reported. All statistical analyses were conducted with IBM SPSS version 24 and a significant level of $p<0.05$ was set.

\section{Results}

\subsection{Perceived Stress}

Total Sample. Table 1 presents mean scores and standard deviations of the perceived stress at the pre-test and the post-test for the total sample and by gender. According to the scoring guidelines for the PSS, scores ranging from 0 13 would be in the low stress category; scores ranging from $14-26$ would be in the moderate stress category; scores ranging from 27 - 40 would be in the high stress category (Cohen et al., 1983). The students' mean score in the PSS at the pre-test was $13.53(\mathrm{SD}=6.36)$ and mean score in the PSS at the post-test was 13.97 ( $\mathrm{SD}=5.19)$, indicating a low end of the moderate level of perceived stress over times. The paired-t test showed no significant changes in the mean score 
Table 1. Descriptive statistics of perceived stress at pre- and the post-test for the total sample and by gender.

\begin{tabular}{ccccc}
\hline Samples & \multicolumn{2}{c}{ Pre-test } & \multicolumn{2}{c}{ Post-test } \\
\hline & $\bar{X}$ & $S D$ & $\bar{X}$ & $S D$ \\
\hline Total sample (38) & 13.53 & 6.36 & 13.97 & 5.19 \\
Female (27) & 13.96 & 6.98 & 13.26 & 5.10 \\
Male (11) & 12.45 & 4.61 & 15.73 & 5.24 \\
\hline
\end{tabular}

from the pre-test to the post-test for the total sample $(t=-0.429, d f=37, p=$ $0.670)$. The total sample's percentage of increase in the perceived stress was $3.25 \%$, a very small increase in the perceived stress.

Gender. Female students' mean scores stayed relatively stable over time (at pre-test: $\bar{X}=13.96, \mathrm{SD}=6.98$; at post-test: $\bar{X}=13.26, \mathrm{SD}=5.10)$. In contrast, the male students' mean scores showed increasing from the low level to the moderate level over times (at pre-test: $\bar{X}=12.45, \mathrm{SD}=4.61$; at post-test: $\bar{X}$ $=15.73, \mathrm{SD}=5.24)$. The results of the Greenhouse-Geyser correction revealed no significant main effect on time and interaction between time and gender $(F=$ $1.323, p=0.258 ; F=3.169, p=0.083)$. Similarly, the results of the ANOVA repeated measures revealed no significant main effect on gender $(F=0.075, p=$ 0.786). However, the female students' percentage of reduction in the perceived stress was $5.30 \%$. In contrast, male students' percentage of increase in the perceived stress was $26.34 \%$, indicating a relatively moderate increase in stress.

\subsection{Anxiety}

Total sample. Table 2 shows mean scores and standard deviations of the anxiety at the pre-test and the post-test for the total sample and by gender. According to the scoring guidelines for the total score of the GAD-7, a score of 5 represents a mild anxiety, 10 indicates moderate anxiety, and 15 represents severe anxiety. The students' average scores showed they had have moderate level of anxiety over times (at pre-test: $\bar{X}=10.95, S D=2.67$; at post-test; $\bar{X}=$ $10.95, S D=2.67)$. The paired-t test found no significant changes in the GAD-7 mean score over time $(t=-1.605, d f=37, p=0.117)$. The results of the effect size showed a small percentage of increase in the anxiety (7.67\%).

Gender. As illustrated in Table 2, female students mean scores at the pre-test and post-test ( $\bar{X}=10.89, S D=2.97 ; \bar{X}=11.56, S D=3.59)$ indicated a moderate level of anxiety over times. Similarly, male students' mean scores $(\bar{X}=$ $11.09, S D=1.87 ; \quad \bar{X}=12.36, S D=3.26)$ showed a moderate level of anxiety over times. The Greenhouse-Geyser correction revealed no significant main effect on time and interaction of time with gender $(F=2.655, p=0.106 ; F=0.269$, $p=0.607)$. Similarly, the ANOVA repeated measures indicated no significant main effect on gender $(F=0.280, p=0.600)$. However, the female students' percentage of increase in the anxiety was $6.15 \%$. In contrast, male students' percentage of increase in the anxiety was $11.45 \%$. 
Table 2. Descriptive statistics of anxiety at pre- and the post-test for the total sample and by gender.

\begin{tabular}{ccccc}
\hline Samples & \multicolumn{2}{c}{ Pre-test } & \multicolumn{2}{c}{ Post-test } \\
\hline Variables & $\bar{X}$ & $S D$ & $\bar{X}$ & $S D$ \\
\hline Total Score of Anxiety & & & & \\
Total Sample & 10.95 & 2.67 & 11.79 & 3.47 \\
Females & 10.89 & 2.97 & 11.56 & 3.59 \\
Males & 11.09 & 1.87 & 12.36 & 3.26 \\
\hline
\end{tabular}

\subsection{Total Sleep Time and Sleep Quality}

Total sample. Table 3 shows mean score and standard deviations of the five sleep variables at the pre-test and the post-test for total sample and by gender. The National Sleep Foundation (2015) recommended that young adults ages 18 25 should sleep for 7 to 9 hours a night, with 6 hours of sleep as the appropriate minimum and 11 as the appropriate maximum to achieve good quality of sleep. As seen in Table 1, the average total sleep times remained in the recommended range. The paired-t tests revealed that the students' average total sleeping time at the post-test was significantly higher than that at the pre-test $(t=2.48, d f=37, p$ $=0.017$ ) with the effect size of $4.82 \%$.

Regarding the sleep quality. As seen in Table 3, the students' sleep efficiency maintained a high efficiency level over times. Regarding sleep disturbances, the students' WASO in minutes, \# of awakenings, and average awakening minutes maintained a very low level over times. The paired t-tests found no significant changes on the four indicators of sleep quality.

Gender. As seen in Table 3, both female students' and male students' average total sleep time were in the recommend range over times. The Greenhouse-Geyser correction yielded a significant main effect of time $(F=4.251, p=0.047, \zeta=$ 1.06), indicating both female and male students' total sleep time significantly increased from pre-test to the post-test. In contrast, the ANOVA repeated measure revealed no significant main effect of gender and the interaction between time and gender $(F=0.155, p=0.696 ; F=0.164, p=0.688$,).

With respect to the four indicators of sleep quality, both female and male students' average sleep efficiency remained a very high level over times. In addition, both female and male students' average awakening minutes stayed very low level over times with a slightly higher in the male students' post-test average wakening minutes. However, female students' average waking minutes and \# of awakening showed a slightly increased pattern from the pre-test to the post-test. In contrast, male students' average waking minutes and \# of awakening slightly decreased over times. The Greenhouse-Geisser correction revealed no significant main effect of time, and the interaction between time and gender. Similarly, the ANOVA repeated measured shown no significant main effect of gender for the four sleep quality variables. 
Table 3. Descriptive statistics of sleep time and quality at pre- and the post-test for the total sample and by gender.

\begin{tabular}{|c|c|c|c|c|}
\hline \multirow{2}{*}{$\begin{array}{l}\text { Samples } \\
\text { Variables }\end{array}$} & \multicolumn{2}{|c|}{ Pre-test } & \multicolumn{2}{|c|}{ Post-test } \\
\hline & $\bar{X}$ & $S D$ & $\bar{X}$ & $S D$ \\
\hline \multicolumn{5}{|l|}{ Sleep Time (min) } \\
\hline Total Sample & 460.22 & 40.844 & 482.58 & 60.281 \\
\hline Females ${ }^{*}$ & 460.84 & 33.122 & 485.55 & 43.462 \\
\hline Males* & 458.71 & 57.588 & 475.29 & 91.935 \\
\hline \multicolumn{5}{|l|}{ Efficiency } \\
\hline Total Sample & $97.59 \%$ & 1.713 & $97.4 \%$ & 1.722 \\
\hline Females & $97.52 \%$ & 1.738 & $97.39 \%$ & 1.621 \\
\hline Males & $97.74 \%$ & 1.723 & $97.42 \%$ & 2.034 \\
\hline \multicolumn{5}{|c|}{ Wake after sleep onset } \\
\hline Total Sample & 12.00 & 10.108 & 12.53 & 9.599 \\
\hline Females & 11.43 & 8.540 & 13.25 & 10.011 \\
\hline Males & 13.39 & 13.617 & 10.77 & 8.691 \\
\hline \multicolumn{5}{|l|}{ \# of Awakenings } \\
\hline Total Sample & 2.84 & 1.705 & 3.03 & 2.062 \\
\hline Females & 2.84 & 1.703 & 3.33 & 2.257 \\
\hline Males & 2.83 & 1.795 & 2.30 & 1.289 \\
\hline \multicolumn{5}{|c|}{ Average Awakening (min) } \\
\hline Total Sample & 3.98 & 2.336 & 3.98 & 1.785 \\
\hline Females & 4.02 & 2.326 & 3.74 & 1.611 \\
\hline Males & 3.86 & 2.471 & 4.57 & 2.122 \\
\hline
\end{tabular}

Note. ${ }^{*}$ indicating a significant change from the pre-test to the post-test at $p<0.01$ level. ${ }^{*}$ indicating a significant change from the pre-test to the post-test as $p<0.05$ level.

\section{Discussion}

This pilot study was central to examining the effects of the 16-week Tai Chi classes on the perceived stress, anxiety, and sleep time and quality in college students and its effects on gender. We found that the students maintained their low end of the moderate level in perceived stress and anxiety from the beginning of the semester (pre-test) to the end of the semester (post-test). It is important to note that in the present study, male students' percentage of increases in perceived-stress and anxiety was relatively moderate, compared to female students (very small percentage of increases). Our findings were consistent with the study findings by Zheng et al. (2015). They found that the intervention students who participated in five, one-hour Tai Chi exercises sessions weekly for 12 weeks did not significantly improve their self-reported stress levels. However, Zheng et al. (2015) did not report whether the students' perceived stress level at the pre-test and the post-test was classified into a low level, a moderate level, or high level. 
Stress is a part of our daily life experiences (Henderson, Snyder, Gupta, \& Banich, 2012; Pidgeon, McGrath, Magya, Stapleton, \& Lo, 2014). Stress itself has no harmful effects on people's cognitive, emotional, and physical functions. Stress can be either good stress or bad stress depending on levels of stress, types of stressors, how to respond to the stressor. The goal-oriented stressor could be a fuel to sharpen our mind, activate our cognitive functions, and vitalize our body energies to strive for accomplishing the goal when we choose to respond to the stressor positively (Henderson et al., 2012; Jaret, 2015). Throughout the semester, the college students are facing and responding to goal-oriented stressors such as academic testing, conducting and presenting the class-related project, applying for internships, scholarships, and summer jobs, etc. In addition, they are experiencing other types of stressors such as social relationship with their boy or girl friend, roommates, classmates, team members, club members, and/or friends. Especially, at the end of the semester, college students are facing increased academic and emotional pressures because they are busy at taking final examines, writing and presenting the course-related projects as well as planning trips to home and so on. Given the unique situations of the university, the levels of the students' perceived stress and anxiety at the end of the semester are more likely to be relatively high, compared to the beginning of the semester. Mounting evidence shows that college students who experience high levels of stress and anxiety are more likely to lead to mental health problems such as depression (Pidgeon et al., 2014). However, in our study, the students who participate in the semester-long Tai Chi classes did not increase their stress and anxiety from a moderate level to a high level even though they were facing and experiencing more increased academic stressors at the end of the semester. Furthermore, recent studies have found that experiencing a moderate level of manageable stress can improve mental alertness and memory and boost cognitive performance (Henderson et al., 2012; Jaret, 2015).

It is worthy to note that the pre-test occurred at the beginning of the semester in which students' academic pressures were relatively low. In contrast, the post-test occurred at the end of the semester in which students' academic and social emotional pressures were relatively high. However, after participating in the 16-week semester Tai Chi classes, the students' perceived stress level and anxiety did not significantly increase even though they were in the final exam weeks during the end of the semester. It, also, should be noted that the participating students did not have any previous experience in learning and practicing Tai Chi prior to taking the semester-long Tai Chi class. The 16-week Tai Chi course was primarily designed for students to learn each form of Tai Chi and to be able to perform the entire Tai Chi routines (16-forms of Tai Chi) by the end of the semester. Accordingly, the instructor focused on helping the students learn a new Tai Chi form in one lesson and reviewed previous form(s). In other words, the focus of the class was to help the students to learn how to perform each form of Tai Chi, rather than to practice it regularly. This mind-body exercise is a quite different from sports, aerobic and strength exercises college students are typically engaged 
in (Sandlund \& Norlander, 2000). The learning stage of Tai Chi demands on an individual's high level of mental concentration on learning each form due to its eye-hand-arm-trunk-leg-and-feet coordinated movement in a sequential order with slow motion (Wang et al., 2010; Li et al., 2012; Li et al., 2018). Throughout the semester, the students in this study had primarily focused on learning each form of the Tai Chi routines rather than use of Tai Chi as the mindful exercise tool to practice on their regular basis. Although the students did not reach the mindful practice stage (i.e., performing given Tai Chi routines with being mindful of each movement in smooth motion and effortless) yet during the 16-week semester learning Tai Chi, they have maintained the moderate level of stress and anxiety, which are considered to be good and productive for their academic learning and performance. The results of our study were inconsistent with previous studies in which students participating in a semester-long Tai Chi classes significantly improved their perceived stress level and mood (Caldwell et al., 2009; Caldwell et al., 2011; Wang, 2008). However, our study has advanced the literature in examining effects of Tai Chi on psychological well-being in one unique way. Our study indicated that even though students were primarily in the stage of learning Tai Chi, the beneficial effects of students' learning and performing Tai Chi for 90 minutes per week over 16 weeks on maintaining their manageable stress level while facing highly stressful academic demanding situations were apparently evident among the students. This positive benefit obtained from learning and performing the semester-long Tai Chi is extended to the results of sleep time and sleep quality among the students.

In conjunction with maintenance of the moderate level of stress and anxiety, our study found that both female and male students significantly increased their sleep time from the pre-test to the post-test. This result might be related to their unsettled college life in the beginning of the semester. For example, they just moved to the campus after a long summer vocation. There were welcoming events and transition to new classrooms and dorms going on. In addition, they have not seen their classmates/friends for a while due to summer vocation. The fresh, exciting, and social college life at the beginning of the semester could attribute to reducing their typical sleep time. Further, both female and male students in this study maintained a high level of sleep efficiency and a very low level of sleep disturbances (i.e., WASO, average awakening minutes, and \# of awakenings) over the course of the semester. Two studies by Caldwell et al. $(2009,2011)$ reported that the 15-week semester Tai Chi classes generated positively significant effects on improving the college students' sleep quality measured with the Pittsburgh Sleep Quality Index (PSQI). This study extends the previous Tai Chi intervention studies influencing sleep quality with subjective measures to an objective measure of sleep time and sleep quality with ActiGraph activity monitor. This is the first study that examined the effects of the Tai Chi classes on college students' sleep time and quality with the objective measure. This study suggests that students learning and performing a "moving meditation". Tai Chi routines are instrumental to maintaining a good level of sleep quality (i.e., sleep efficiency, 
sleep disturbance) throughout the semester. Further, this study suggests that learning and performing mindful Tai Chi exercises serves as a buffer for college students to prevent them from having sleep deprivation and sleep disturbance problems, especially during the end of the semester.

This study was limited to the use of the pre- and the post-test within subject design. Future studies should be warranted in using either a randomized controlled trial or a quasi-experimental research design to examine the effects of the Tai Chi semester intervention on college students' psychological wellbeing and sleep quality. Another limitation was the Tai Chi intervention strategy and dose used in this study, similar to the four previous Tai Chi studies in college students. The intervention strategy in this study focused on helping students learn Tai Chi routines throughout the semester because these students had no prior learning experiences in Tai Chi routines. Also, the dose of intervention in this study was restrained by having one 90 -min Tai Chi class per week. Given the constrained situations, none of the previous studies and the present study have explored the extent to which using Tai Chi as their regular exercise approach to improve students' abilities for managing their stress and anxiety levels, meeting the recommended sleep hours, and having good sleep quality among college students. For future studies, there is a need for recruiting college students who have learned specific Tai Chi routines (i.e. 12-form, 24-form Tai Chi) to be in the Advanced Tai Chi group, recruiting college students who will learn a specific Tai Chi routine in the upcoming Tai Chi semester classes to be in the Beginning Tai Chi group. Such research design would be helpful for allowing us to find out the effects of regular practicing Tai Chi on students' psychological wellbeing and sleep time and quality. This study did not examine the extent to which students' moderate level of perceived stress and anxiety influenced their academic performance. Future studies may explore the mediating effects of Tai Chi intervention on the relationship of different levels of perceived stress and anxiety with academic performance in college students.

In conclusion, this study suggests that learning and performing Tai Chi is conducive to helping students maintain their manageable level of stress and anxiety while facing and experiencing increased academic-oriented stressors at the end of the semester. Further, participation in the 16-week semester Tai Chi classes was beneficial for the college students to have adequate sleep time and high sleep quality even though they were experiencing a moderate level of stress and anxiety. Further, this study supports the previous findings that having a manageable level of stress and anxiety is not negative to sleep time and quality.

\section{What Does This Article Add?}

Learning while performing Tai Chi for a semester is instrumental in helping college students maintain their manageable level of stress and anxiety and have sufficient sleep time and high sleep quality even though they are facing increased academic-related pressures and stressors at the end of the semester. Due to beneficial effects of Tai Chi on colleges students' psychological well-being, this study 
suggests that providing students with beginning, intermediate, and advance levels of Tai Chi semester classes along with various levels of Tai Chi clubs would best meet college students' needs since they have different levels of Tai Chi learning and practices. Further, this study suggests that future studies may examine both short-term and long-term effects of regular performance of Tai Chi on perceived stress and anxiety as well as sleep time and quality among college students.

\section{Funding}

This research did not receive any specific grants from funding agencies in the public, commercial, or no-profit sectors.

\section{IRB Approval}

This study protocols were approved by the University Institutional Review Board-Health Sciences and Behavioral Sciences (IRB-HSBS) (HUM00102146). Students were assured confidentiality and completed consent forms voluntarily before the beginning of the study.

\section{Acknowledgements}

We are thankful for the students at East China University of Political Science and Law to participate in this study.

\section{Conflicts of Interest}

All authors declared no potential conflicts of interest with respect to the research, authorship, and/or publication of this article.

\section{References}

American College Health Association (2018). American College Health Association, National College Health Assessment II: Reference Group Executive Summary Spring 2018. Silver Spring, MD: American College Health Association.

Caldwell, K., Emery, L., Harrison, M., \& Greeson, J. (2011). Changes in Mindfulness, Well Being, and Sleep Quality in College Students through Taijiquan Courses: A Cohort Control Study. The Journal of Alternative and Complementary Medicine, 17, 931-938. https://doi.org/10.1089/acm.2010.0645

Caldwell, K., Harrison, M., Adams, M., \& Triplett, N. T. (2009). Effect of Pilates and Taijiquan Training on Self-Efficacy, Sleep Quality, Mood, and Physical Performance of College Students. Journal of Bodywork and Movement Therapies, 13, 155-163.

https://doi.org/10.1016/j.jbmt.2007.12.001

Chung, K. F., \& Cheung, M. M. (2008). Sleep-Wake Patterns and Sleep Disturbance among Hong Kong Chinese Adolescents. Sleep, 31, 185-194. https://doi.org/10.1093/sleep/31.2.185

Cohen, S., Kamarck, T., \& Mermelstein, R. (1983). A Global Measure of Perceived Stress. Journal of Health and Social Behavior, 24, 385-396. https://doi.org/10.2307/2136404

He, X. Y., Li, C. B., Qian, J., Cui, H. S., \& Wu, W. Y. (2010). Reliability and Validity of a Generalized Anxiety Disorder Scale in General Hospital Outpatients. Shanghai Archives Psychiatry, 22, 200-203.

Henderson, R. K., Snyder, H. R., Gupta, T., \& Banich, M. T. (2012). When Does Stress 
Help or Harm? The Effects of Stress Controllability and Subjective Stress Response on Stroop Performance. Frontiers in Psychology, 3, 179.

https://doi.org/10.3389/fpsyg.2012.00179

Jaret, P. (2015). A Surprising Benefit of Stress: A UG Berkeley Researcher Is Discovering the Differences between Good and Bad Stress. https://greatergood.berkeley.edu/article/item/the_surprising_benefits_of_stress

Lee, J. M., Byun, W., Keill, A., Dinkel, D., \& Seo, Y. (2018). Comparison of Wearable Trackers' Ability to Estimate Sleep. International Journal of Environmental Research and Public Health, 15, 1265. https://doi.org/10.3390/ijerph15061265

Leung, D. Y., Lam, T. H., \& Chan, S. S. (2010). Three Versions of Perceived Stress Scale: Validation in a Sample of Chinese Cardiac Patients Who Smoke. BMC Public Health, 10, Article No. 513. http://www.biomedcentral.com/1471-2458/10/513 https://doi.org/10.1186/1471-2458-10-513

Li, F., Harmer, P., Fitzgerald, K., Batya, S. S. et al. (2012). Tai Chi and Postural Stability in Patients with Parkinson's Disease. The New England Journal of Medicine, 266, 511-519. https://doi.org/10.1056/NEJMoa1107911

Li, F., Harmer, P., Fitzgerald, K., Winters-Stone, K. et al. (2018). Effectiveness of a Therapeutic Taijiquan Intervention vs. a Multimodal Exercise Intervention on Prevent Falls among Older Adults at High Risk of Falling: A Randomized Clinical Trial. JAMA Internal Medicine, 178, 1301-1310. https://doi.org/10.1001/jamainternmed.2018.3915

Lund, H. G., Reider, B. D., Whiting, A. B., \& Richard J. R. (2010). Sleep Patterns and Predictors of Disturbed Sleep in a Large Population of College Students. Journal of Adolescent Health, 46, 124-132. https://doi.org/10.1016/j.jadohealth.2009.06.016

Pidgeon, A. M., McGrath, S., Magya, H. B., Stapleton, P., \& Lo, B. C. Y. (2014). Psychosocial Moderators of Perceived Stress, Anxiety, and Depression in University Students: An International Study. Open Journal of Social Sciences, 2, 23-31. https://doi.org/10.4236/jss.2014.211004

Sandlund, E. S., \& Norlander, T. (2000). The Effects of Tai Chi Chuan Relation and Exercise on Stress Responses and Well-Being: An Overview of Research. International Journal of Stress Management, 7, 139-149. https://doi.org/10.1023/A:1009536319034

Spitzer, R. L., Kroenke, K., Williams, J. B., \& Lowe, B. (2006). A Brief Measure for Assessing Generalized Anxiety Disorder: The GAD-7. Archives of Internal Medicine, 166, 1092-1097. https://doi.org/10.1001/archinte.166.10.1092

Wang, C., Bannuru, R., Ramel, Kupelnick, B., Scott, B., \& Schmid, C. H. (2010). Tai Chi on Psychological Well-Being: Systematic Review and Meta-Analysis. BMC Complementary and Alternative Medicine, 10, Article No. 23.

https://doi.org/10.1186/1472-6882-10-23

Wang, Y. T. (2008). Tai Chi Exercise and the Improvement of Mental and Physical Health among College Students. In Y. Hong (Ed.), Tai Chi Quan: State of the Art in International Research (Medicine and Sport Science, Vol. 52) (pp. 135-145). Basel: S. Karger. https://doi.org/10.1159/000134294

Wang, Y. T., Taylor, L., Pearl, M., \& Chang, L. S. (2004). Effects of Tai Chi Exercise on Physical and Mental Health of College Students. The American Journal of Chinese Medicine, 32, 453-459. https://doi.org/10.1142/S0192415X04002107

Zheng, G., Lan, X., Li, M., Fang, Q. et al. (2015). Effectiveness of Tai Chi on Physical and Psychological Health of College Students: Results of a Randomized Controlled Trial. PLOS ONE, 10, e0132605. https://doi.org/10.1371/journal.pone.0132605

Zheng, G., Li, S., Huang, M., Liu, F., Tao, J., \& Chen, L. (2015). The Effect of Tai Chi Training on Cardiorespiratory Fitness in Healthy Adults: A Systematic Review and Meta-Analysis. PLoS ONE, 10, e0117360. https://doi.org/10.1371/journal.pone.0117360 